\title{
Numerical solution of subsonic and transonic flows in 2D and 3D
}

Jaroslav Huml ${ }^{1, \mathrm{a}}$ and Karel Kozel ${ }^{1}$

${ }^{1}$ Czech Technical University in Prague, Faculty of Mechanical Engineering

\begin{abstract}
This work deals with a numerical simulation of 2D and 3D inviscid and laminar compressible flows around a DCA $18 \%$ profile. Numerical results were achieved on non-orthogonal structured grids by the authors' in-home code with an implemented FVM multistage Runge-Kutta method and an artificial dissipation. The results are discussed and compared with other similar ones (e.g. the results by G. S. Deiwert).
\end{abstract}

\section{Introduction}

The authors deal with the development of their own inhome codes that could be applied in general for the simulation of any flow, i.e. an inviscid and a viscous (both laminar and turbulent) flow, using one or more FVM schemes. In this case the code includes a multistage Runge-Kutta method with a Jameson artificial dissipation and has been successfully tested on the simulation of inviscid and laminar flows in a few test cases, i. e. flows in the GAMM channel and in a DCA $8 \%$ cascade. Based on the previous successfull use (see i. e. [4] or [5]) authors have decided to apply it for numerical solutions of inviscid and laminar flows around a DCA $18 \%$ profile in $2 \mathrm{D}$ and $3 \mathrm{D}$, and to verify its general application.

\section{Mathematical models}

\subsection{Navier-Stokes equations}

A general two-dimensional flow of a compressible laminar fluid (the authors assumed that the flowing medium was a calorically perfect gas - the Newtonian fluid.) is described by the system of Navier-Stokes equations

$$
\mathbf{W}_{t}+\mathbf{F}_{x}+\mathbf{G}_{y}=\mathbf{R}_{x}+\mathbf{S}_{y}
$$

where $^{2}$

$$
\begin{aligned}
\mathbf{W} & =(\rho, \rho u, \rho v, e)^{T}, \\
\mathbf{F} & =\left(\rho u, \rho u^{2}+p, \rho u v,(e+p) u\right)^{T}, \\
\mathbf{G} & =\left(\rho v, \rho u v, \rho v^{2}+p,(e+p) v\right)^{T},
\end{aligned}
$$

\footnotetext{
a e-mail: jar.huml@gmail.com

$2 \rho$ denotes density of the fluid; $(u, v, w)$ are components of the local velocity respectively in the direction of axis $x, y, z ; p$ is pressure (given by an equation of state); $e$ denotes density of total energy per a unit volume; $\tau_{i j}$ represents shear stress (assuming Stokes Law for mono-atomic gases); $q_{j}$ is heat flux (given by Fourier's Law assuming Mayer's formula); $\mu$ represents dynamical viscosity; $\gamma$ denotes the isentropic exponent (equals 1.4 for mono-atomic gases - air); and $P r$ is laminar Prandtl number (equals 0.9 ).
}

$$
\begin{aligned}
\mathbf{R} & =\left(0, \tau_{x x}, \tau_{x y}, u \tau_{x x}+v \tau_{x y}-q_{x}\right)^{T}, \\
\mathbf{S} & =\left(0, \tau_{x y}, \tau_{y y}, u \tau_{x y}+v \tau_{y y}-q_{y}\right)^{T}
\end{aligned}
$$

and

$$
\begin{aligned}
p & =(\gamma-1)\left[e-\frac{1}{2}\left(u^{2}+v^{2}\right)\right], \\
\tau_{i j} & =\mu\left(\frac{\partial u_{i}}{\partial x_{j}}+\frac{\partial u_{j}}{\partial x_{i}}\right)-\frac{1}{3} \delta_{i j} \frac{\partial u_{k}}{\partial x_{k}}, \\
q_{j} & =-\frac{\gamma}{\gamma-1} \frac{\mu}{\operatorname{Pr}} \frac{\partial}{\partial x_{j}}\left(\frac{p}{\rho}\right) .
\end{aligned}
$$

\subsection{Euler equations}

Assuming an inviscid fluid ( $\mu=0$ in viscous fluxes $\mathbf{R}, \mathbf{S}$ ), the system of Euler equations is used instead of the system of Navier-Stokes equations

$$
\mathbf{W}_{t}+\mathbf{F}_{x}+\mathbf{G}_{y}=0
$$

or in case of 3D simulation

$$
\mathbf{W}_{t}+\mathbf{F}_{x}+\mathbf{G}_{y}+\mathbf{H}_{z}=0 .
$$

where

$$
\begin{aligned}
\mathbf{W} & =(\rho, \rho u, \rho v, \rho w, e)^{T} \\
\mathbf{F} & =\left(\rho u, \rho u^{2}+p, \rho u v, \rho u w,(e+p) u\right)^{T}, \\
\mathbf{G} & =\left(\rho v, \rho u v, \rho v^{2}+p, \rho v w,(e+p) v\right)^{T}, \\
\mathbf{H} & =\left(\rho w, \rho u v, \rho u w, \rho u^{2}+p,(e+p) u\right)^{T}, \\
p & =(\gamma-1)\left[e-\frac{1}{2}\left(u^{2}+v^{2}+w^{2}\right)\right],
\end{aligned}
$$

\subsection{Transformation to the dimensionless form}

All variables were considered dimensionless and inflow variables $(.)_{\infty}$ were used as reference variables for the transformation of the equations to dimensionless form (e.g. $\bar{\rho}=$ $\left.\rho / \rho_{\infty}\right)$. Thus after the transformation the system of Euler equations is written in the same form, but the transformed 
system of Navier-Stokes equations ${ }^{3}$ (7) is a little different from aforementioned (1)

$$
\mathbf{W}_{t}+\mathbf{F}_{x}+\mathbf{G}_{y}=\frac{M a_{\infty}}{R e_{\infty}}\left(\mathbf{R}_{x}+\mathbf{S}_{y}\right) .
$$

\section{Numerical method and scheme}

The FVM numerical scheme - the multistage Runge-Kutta method extended by including Jameson's artificial dissipation to improve the stability of the method ${ }^{4}$ - was applied to the cell centered form on non-orthogonal structured grids of quadrilateral or hexahedral cells $D^{5}$ for the simulation of the flows mentioned previously.

- multistage Runge-Kutta method $(R K)$

$$
\begin{aligned}
\operatorname{Res} \mathbf{W}_{i j}^{(r)}= & \frac{1}{\left|D_{i j}\right|} \sum_{k=1}^{4}\left(\tilde{\mathbf{F}}_{k}^{r} \Delta y_{k}-\tilde{\mathbf{G}}_{k}^{r} \Delta x_{k}\right), \\
\mathbf{W}_{i j}^{(0)}= & \mathbf{W}_{i j k}^{n}, \\
\mathbf{W}_{i j k}^{(r+1)}= & \mathbf{W}_{i j k}^{(0)}-\alpha_{r} \Delta t \operatorname{Res} \mathbf{W}_{i j k}^{(r)}+A D\left(\mathbf{W}_{i j k}^{n}\right), \\
\mathbf{W}_{i j k}^{n+1}= & \mathbf{W}_{i j k}^{(3)}, \\
& \alpha_{0,1}=0.5, \alpha_{2}=1, r=0,1,2 .
\end{aligned}
$$

- Jameson's artificial dissipation $(A D)$

$$
\begin{aligned}
A D\left(\mathbf{W}_{i, j}^{n}\right) & =C_{1} \psi_{1}\left(\mathbf{W}_{i-1, j}^{n}-2 \mathbf{W}_{i, j}^{n}+\mathbf{W}_{i+1, j}^{n}\right) \\
& +C_{2} \psi_{2}\left(\mathbf{W}_{i, j-1}^{n}-2 \mathbf{W}_{i, j}^{n}+\mathbf{W}_{i, j+1}^{n}\right),
\end{aligned}
$$

where

$$
\begin{aligned}
& \psi_{1}=\frac{\left|p_{i-1, j}^{n}-2 p_{i, j}^{n}+p_{i+1, j}^{n}\right|}{\left|p_{i-1, j}^{n}\right|+\left|p_{i, j}^{n}\right|+\left|p_{i+1, j}^{n}\right|}, \\
& \psi_{2}=\frac{\left|p_{i, j-1}^{n}-2 p_{i, j}^{n}+p_{i, j+1}^{n}\right|}{\left|p_{i, j-1}^{n}\right|+\left|p_{i, j}^{n}\right|+\left|p_{i, j+1}^{n}\right|} .
\end{aligned}
$$

Numerical approximations $\tilde{\mathbf{F}}, \tilde{\mathbf{G}}$ of convective terms $\mathbf{F}, \mathbf{G}$ were considered in the forward form of the first order of accuracy. Numerical approximations $\tilde{\mathbf{R}}, \tilde{\mathbf{S}}$ of dissipative terms $\mathbf{R}, \mathbf{S}$ were approximated in the central form of the second order of accuracy and by using dual cells and applying Green's formula.

The time step $\Delta t$ defined by the stability criterion was computed by

$$
\Delta t \leq \frac{C F L}{\frac{|u|+a}{\Delta x}+\frac{|v|+a}{\Delta y}+2 \frac{M a_{\infty}}{R e_{\infty}}\left(\frac{1}{\Delta x^{2}}+\frac{1}{\Delta y^{2}}\right)} \cdot
$$

$3 M a_{\infty}, R e_{\infty}$ is inlet Mach and Reynolds number.

4 The scheme and the artificial dissipation are written for a 2D flow, but their 3D forms look similar.

${ }^{5}|D|$ represents the volume of the cell.

$6 C F L=2$ for the multi-stage Runge-Kutta method chosen and $a$ is the sound speed.

\section{Formulation of the problems}

The authors took into account the numerical simulations of 2D inviscid and laminar compressible flows around the DCA $18 \%$ profile and around its 3D modification (a simple extension into 3D and a Swept Wing modification). The outlines of the computational domain are shown in figures 1 and 2 . The left and right outlines $(\overline{A D}, \overline{B C})$ and surfaces $(\square A D L I, \square B C K J)$ represent an inlet and outlet of the domain respectively. The bottom and top outlines $(\overline{A B}, \overline{C D})$ and surfaces ( $\square A B J I, \square D C K L)$ are so called free walls, where the same condition was considered similar to at intlet. The symmetry line and plane are divided into two straight lines $(\overline{E F}, \overline{G H})$ and surfaces $(\square E F N M, \square G H P O)$ respectively, into the parts of the boundary where a symmetry condition was applied. And finally two circular curves between the points $(F, G)$ and two curved surfaces $(\square F G O N)$ represent the bottom/top part of DCA $18 \%$ profile - here were prescribed boundary conditions for a solid wall according to the mentioned type of flow (an inviscid or a viscous flow).

In the case of the Swept Wing modification the relative thickness and chord are linearly changed depending on the $z$-coordinate between $(10 \%, 18 \%)$ and $(0.5,1.0)$ respectively.

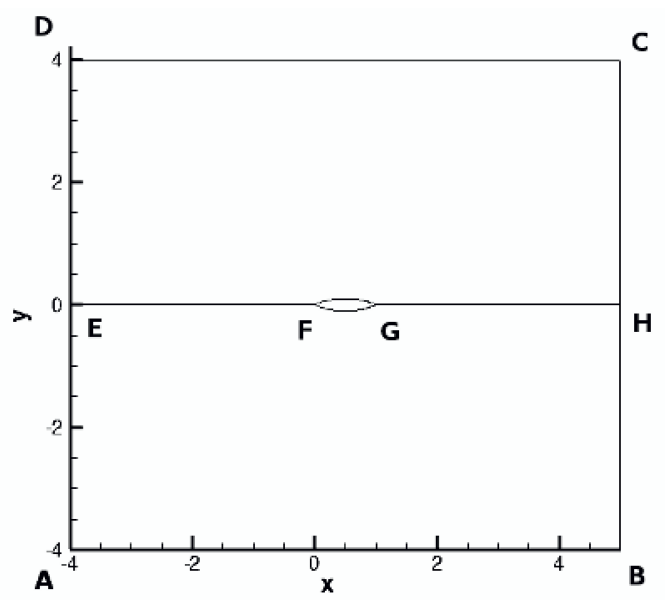

Fig. 1. Geometry of the DCA $18 \%$ profile in 2D.

The $2 \mathrm{D}$ region and 3D region were covered by three different non-orthogonal structured grids, quadrilateral meshes with $160 \times 160$ cells (see figures 3 and 4 ) and hexahedral grid with $160 \times 160 \times 1$ or $160 \times 160 \times 10$ cells (see figure 5), where 60 cells were distributed over the DCA $18 \%$ profile in the $x$-direction. The authors made also such a convenient refinement of the mesh around the profile in the $y$-direction for a better detection of viscosity influence in the case of the laminar flows (see figures 4$)$. The smallest spacing was equalled $2 /\left(3 \sqrt{R e_{\infty}}\right)$.

\subsection{Boundary conditions}

The authors divided the computational domain into two same parts (see figures 1 and 2) - a lower half and an upper 


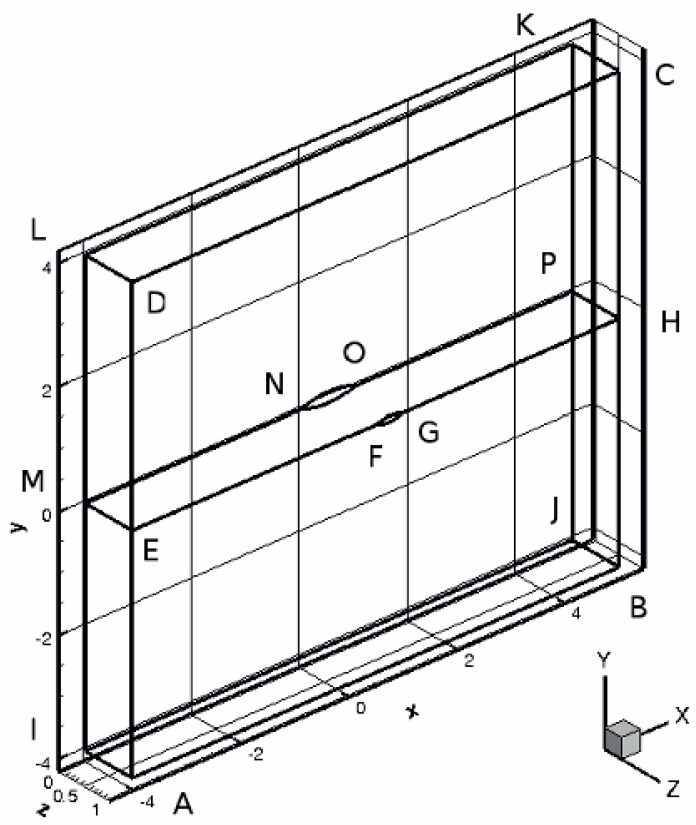

Fig. 2. Geometry of the DCA $18 \%$ profile in 3D - Swept Wing modification.

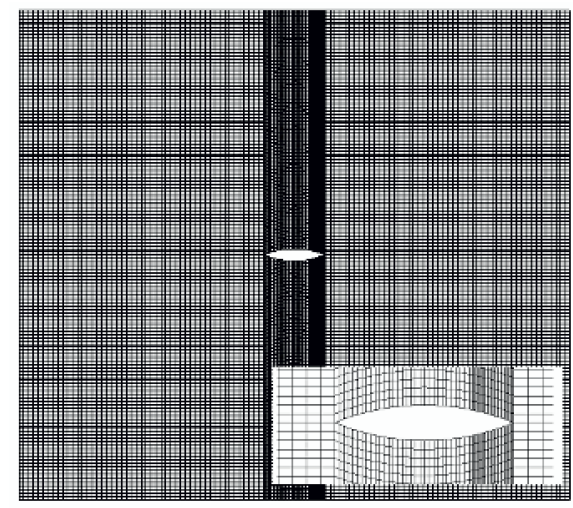

Fig. 3. Mesh for $2 \mathrm{D}$ inviscid flows $-160 \times 160$ cells (grid detail around the profile in the lower right corner).

half - and added virtual cells to these parts from the outside for a better realisation of all the prescribed boundary conditions (see figure 6):

- inlet: $\rho_{1}=1, u_{1}=M a_{1} \cos \alpha, v_{1}=M a_{1} \sin \alpha, p_{1}$ was extrapolated from the flow field and $e_{1}$ was calculated using the equation of state.

- outlet: $p_{2}$ was prescribed and the other components were extrapolated from the flow field or calculated.

- solid wall: velocity components were prescribed so that the sum of velocity vectors equals zero (viscous flows $u=v=0$ ) or equals zero in their tangential component (inviscid flows $-(u, v) \mathbf{n}=0)$.

- free wall: the value of a variable in the cell at the bottom/top part of the boundary corresponds to the value at the inlet.

- symmetry: the value of a variable in the top cells of the lower half corresponds to the value in the bottom cells of the upper half and vice versa.

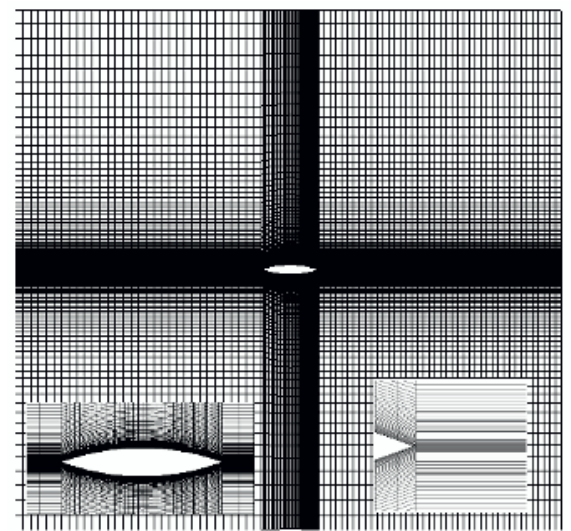

Fig. 4. Mesh for 2D laminar flows $-160 \times 160$ cells (grid detail around the profile in the lower left corner and grid detail near the trailing edge in the lower right corner).

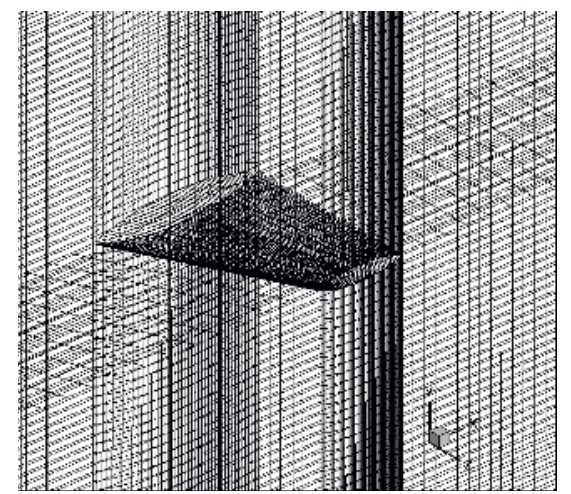

Fig. 5. Mesh for 3D inviscid flows $-160 \times 160 \times 10$ cells (grid detail of swept wing).

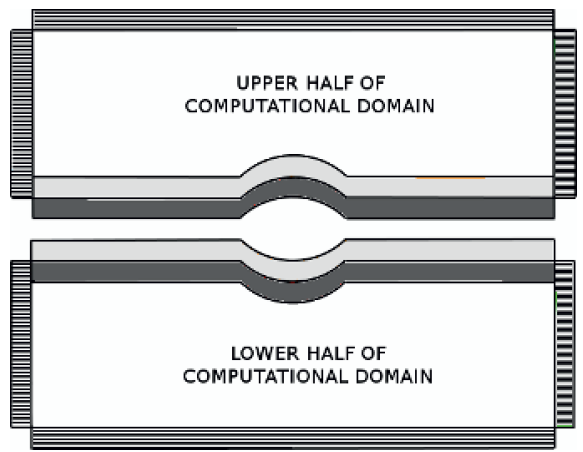

Fig. 6. Application of boundary conditions - inlet and free wall b.c. (stripes), outlet b.c. (fine stripes) and symmetry/solid wall b.c. (light and dark grey).

\section{Numerical results and discussion}

As mentioned above, the authors dealt with the numerical simulation of 2D and 3D inviscid and laminar transonic flows around the DCA $18 \%$ profile and applied the multistage Runge-Kutta method with Jameson's additional artificial viscosity on three non-orthogonal structured grids with $160 \times 160$ cells and with $160 \times 160 \times 1$ or $160 \times 160 \times$ 10 cells for $2 \mathrm{D}$ and $3 \mathrm{D}$ case respectively (see figures 3 5). The authors considered a value of inlet Mach number $M a_{\infty}=0.775$ and few different values of Reynolds num- 
bers $R e_{\infty} \in\left(10^{5}, 10^{7}\right)$ (in laminar flows) and angles of attack $\alpha \in\left(-3^{\circ}, 3^{\circ}\right)$.

All the numerical results are presented by using Mach number isolines and isosurfaces, the results for 2D inviscid flows are shown in figures 7 and 8 and for 3D inviscid flows are printed in figures 12 (the simple extension into 3D) and 13 (the Swept Wing modification).

The authors have used the results by G. S. Deiwert [1] for comparison of $2 \mathrm{D}$ results and do realize that this comparison is only informative since our results are not turbulent in contrast to Deiwert's ones. From the comparison of the results it follows that, despite the fact that the authors used a larger number of cells in the $x$ - and $y$-direction than Deiwert, the minimum values of the pressure coefficient $\left(c_{p} \approx-0.9\right.$, see figure 10$)$ are almost half than the values by Deiwert $\left(c_{p} \approx-1.4\right)$ while the $x$-position of the minimum pressure coefficient is almost the same $(x \approx 0.7)$.

In the case of $3 \mathrm{D}$ flows the authors did not find any appropriate comparable resuts, nevertheless the $3 \mathrm{D}$ results were compared at least with the results of compressible flows in a 3D modification of the GAMM channel (an upper half Swept Wing, see [4] or [5]). Based on the comparison all the numerical results evince expected characters of the mentioned flow types.

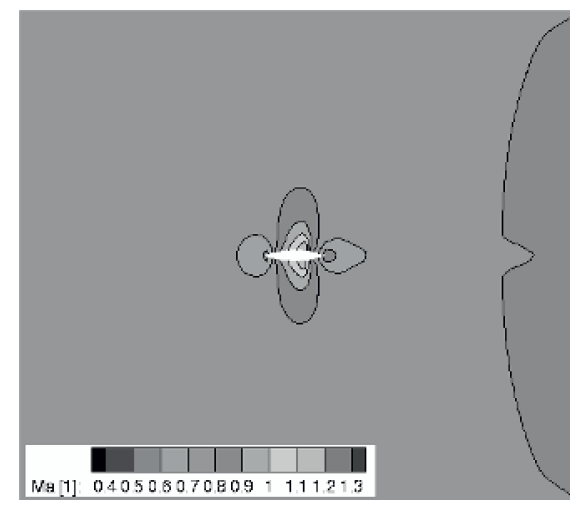

Fig. 7. Inviscid compressible flow around the DCA $18 \%$ profile at $M a_{\infty}=0.775$ and $\alpha_{\infty}=0^{\circ}$ : Mach number isolines $-R K$ scheme, mesh with $160 \times 160$ cells.

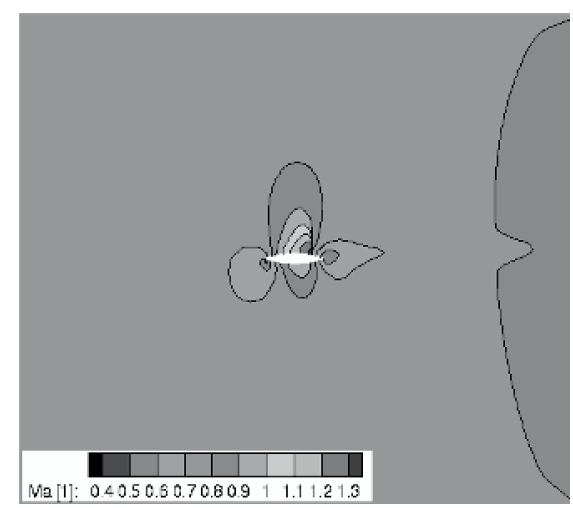

Fig. 8. Inviscid compressible flow around the DCA $18 \%$ profile at $M a_{\infty}=0.775$ and $\alpha_{\infty}=3^{\circ}$ : Mach number isolines $-R K$ scheme, mesh with $160 \times 160$ cells.

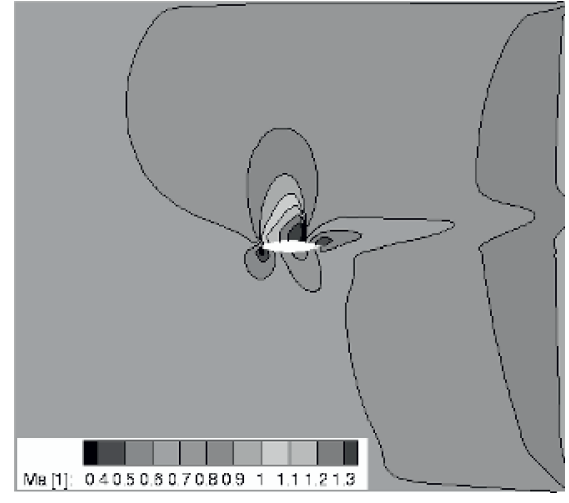

Fig. 9. Inviscid compressible flow around the DCA $18 \%$ profile at $M a_{\infty}=0.775$ and $\alpha_{\infty}=10^{\circ}$ : Mach number isolines $-R K$ scheme, mesh with $160 \times 160$ cells.

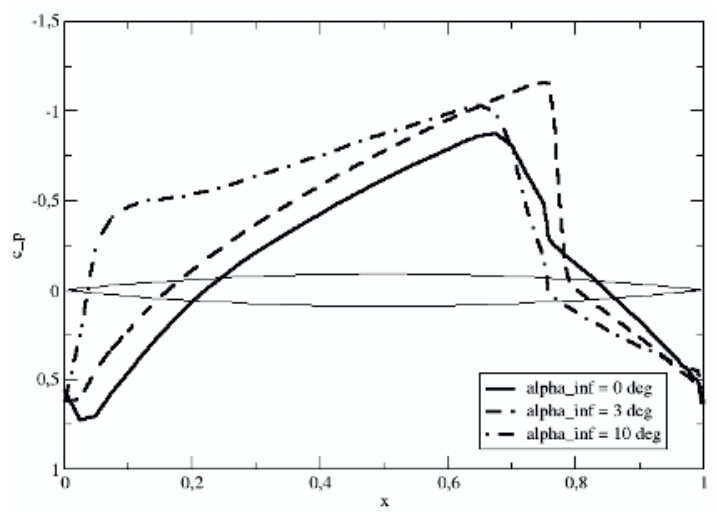

Fig. 10. Pressure coefficient distribution over the DCA $18 \%$ profile at $M a_{\infty}=0.775$

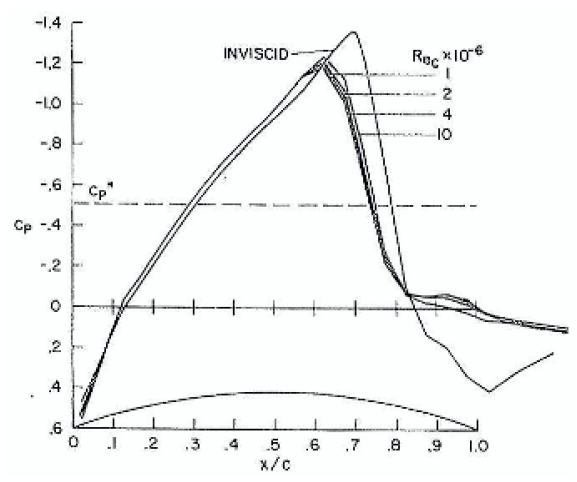

Fig. 11. Pressure coefficient distribution over the DCA $18 \%$ profile at $M a_{\infty}=0.775$ (source [1]). 


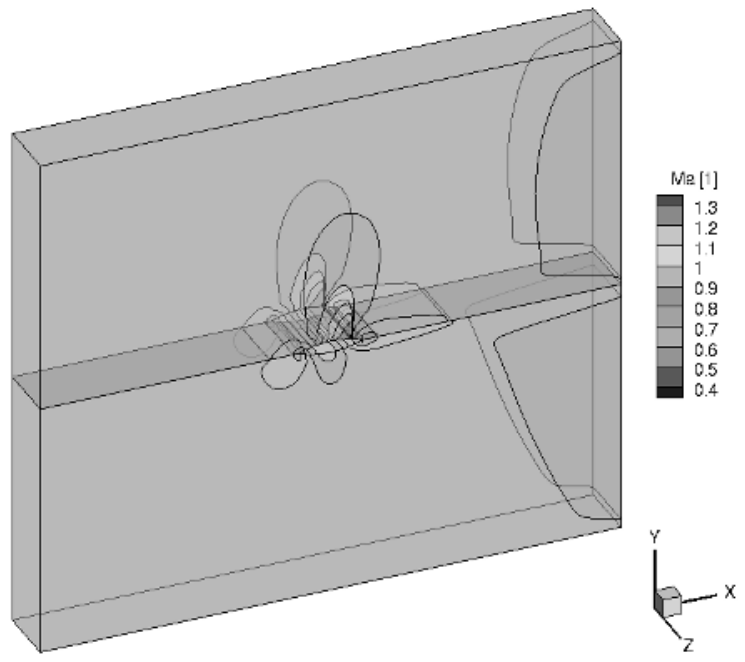

Fig. 12. Inviscid compressible flow around the DCA $18 \%$ profile at $M a_{\infty}=0.775$ and $\alpha_{\infty}=3^{\circ}$ : Mach number isolines $-R K$ scheme, mesh with $160 \times 160 \times 1$ cells.

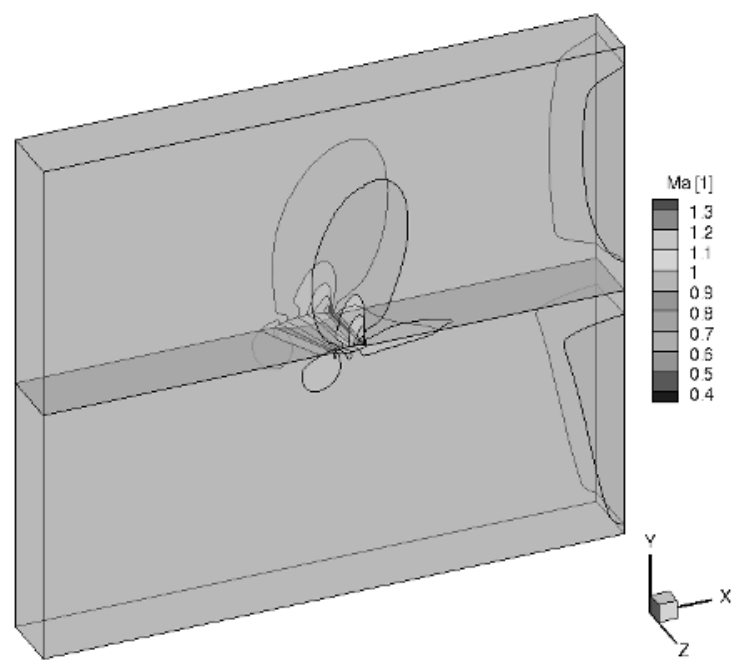

Fig. 13. Inviscid compressible flow around the DCA $18 \%$ profile at $M a_{\infty}=0.775$ and $\alpha_{\infty}=3^{\circ}$ : Mach number isolines $-R K$ scheme, mesh with $160 \times 160 \times 10$ cells.

\section{Conclusions}

This article presents some results achieved by using the inhome code with the implemented FVM multistage RungeKutta method and Jameson's artificial dissipation for the simulation of a 2D and 3D transonic flows of the inviscid and laminar compressible fluid around the DCA $18 \%$ profile. All the numerical results show the expected characters of mentioned flow types.

\section{Acknowledgements}

This work was partly supported by grants No. P101/10/1329 and P101/12/1271 of the Grant Agency of the Czech Republic, grant No. IAA200760801 of the Grant Agency of the Academy of Science of the Czech Republic.

\section{References}

1. G. S. Deiwert, the Fourth International Conference on Numerical Methods in Fluid Dynamics 35, 132-137 (1975)

2. R. Dvořák, Transonic Flows (1986) [in Czech]

3. R. Dvořák, K. Kozel, Mathematical modelling in aerodynamics (1996) [in Czech]

4. J. Huml, K. Kozel, J. Př́hoda, 11th conference on Power System Engineering, Thermodynamics $\mathcal{E}$ Fluid Flow 2012, 73-76 (2012)

5. J. Trefilík, J. Huml, K. Kozel, J. Příhoda, CzechJapanese Seminar in Applied Mathematics 2010, 94-107 (2010) 